PROCEEDINGS OF THE

AMERICAN MATHEMATICAL SOCIETY

Volume 137, Number 12, December 2009, Pages 4307-4312

S 0002-9939(09)10058-8

Article electronically published on August 7, 2009

\title{
ON QUASI-METRIC AND METRIC SPACES
}

\author{
MACIEJ PALUSZYŃSKI AND KRZYSZTOF STEMPAK
}

(Communicated by Nigel J. Kalton)

\begin{abstract}
Given a space $X$ with a quasi-metric $\rho$ it is known that the socalled $p$-chain approach can be used to produce a metric in $X$ equivalent to $\rho^{p}$ for some $0<p \leq 1$, hence also a quasi-metric $\tilde{\rho}$ equivalent to $\rho$ with better properties. We refine this result and obtain an exponent $p$ which is, in general, optimal.
\end{abstract}

\section{INTRODUCTION}

A quasi-metric on a nonempty set $X$ is a mapping $\rho: X \times X \rightarrow[0, \infty)$ which satisfies the following conditions:

(i) for every $x, y \in X, \rho(x, y)=0$ if and only if $x=y$;

(ii) for every $x, y \in X, \rho(x, y)=\rho(y, x)$;

(iii) there is a constant $K \geq 1$ such that for every $x, y, z \in X$,

$$
\rho(x, y) \leq K(\rho(x, z)+\rho(z, y)) .
$$

The pair $(X, \rho)$ is then called a quasi-metric space; if $K=1$, then $\rho$ is a metric and $(X, \rho)$ is a metric space.

Condition (iii) can be replaced by

(iii)' there is a constant $K_{o} \geq 1$ such that for every $x, y, z \in X$,

$$
\rho(x, y) \leq K_{o} \max \{\rho(x, z), \rho(z, y)\},
$$

which is equivalent to (iii) if we do not care about constants entering into both conditions, but is slightly more restrictive than (iii) if we do: (iii)' implies (iii) with $K=K_{o}$, while (iii) implies (iii)' with $K_{o}=2 K$. It should be pointed out that in the area of general topology a quasi-metric is often understood as a mapping $\rho$ which violates the symmetry condition (ii) rather than the triangle inequality (i.e. (i) and (iii) with $K=1$ are assumed to hold). In the present paper we adhere to the definition given above.

Two quasi-metrics $\rho_{1}$ and $\rho_{2}$ on $X$ are said to be equivalent if $c^{-1} \rho_{2}(x, y) \leq$ $\rho_{1}(x, y) \leq c \rho_{2}(x, y)$ with some $c>0$ independent of $x, y \in X$.

Macías and Segovia proved [8, Theorem 2] that given a quasi-norm $\rho$ it is possible to construct a quasi-metric $\rho^{\prime}$ equivalent to $\rho$ and such that the quasi-metric balls related to $\rho^{\prime}$ are open in the topology $\mathcal{F}_{\rho^{\prime}}=\mathcal{F}_{\rho}$; see Section 2 for the definition of

Received by the editors January 18, 2009, and, in revised form, May 12, 2009.

2000 Mathematics Subject Classification. Primary 54E35; Secondary 54E15.

Key words and phrases. Quasi-metric, p-chain approach.

The authors' research was supported in part by grants KBN \#1P03A03029 and MNiSW \#N201 $05432 / 4285$, respectively.

(C)2009 American Mathematical Society 
$\mathcal{F}_{\rho}$. More precisely, they proved that for a given $\rho$ there exist a quasi-metric $\rho^{\prime}$, a number $0<\alpha<1$ and $C>0$ such that $\rho^{\prime}$ is equivalent to $\rho$ and

$$
\left|\rho^{\prime}(x, z)-\rho^{\prime}(y, z)\right| \leq C \rho^{\prime}(x, y)^{\alpha}\left(\max \left\{\rho^{\prime}(x, z), \rho^{\prime}(y, z)\right\}\right)^{1-\alpha}, \quad x, y, z \in X .
$$

A direct computation then shows that the above inequality indeed implies the fact that the quasi-metric balls related to $\rho^{\prime}$ are open in $\mathcal{F}_{\rho^{\prime}}=\mathcal{F}_{\rho}$.

Then Aimar, Iaffei and Nitti 1 furnished a direct proof of the aforementioned result. In [1] a construction of Frink [5] was adapted to produce from a given $\rho$, with appropriately chosen $p, 0<p<1$, the metric $d_{p}$ (see (2.1)) equivalent to $\rho^{p}$. The question of finding some $p$ so that $\rho^{p}$ is equivalent to a metric is also discussed in 6, p. 110]. The analogous result for quasi-normed spaces is called the Aoki-Rolewicz theorem; see [2], [10, and also [7] and [9].

The aim of this paper is to refine the result of [1; see the comments at the end of Section 2 that explain the refinement. Also, we take the opportunity to furnish an example and make some remarks related to the question, when is it that quasimetric balls related to a given quasi-metric $\rho$ are open sets in the topology induced in $X$ by $\rho$.

Quasi-metric spaces are naturally involved in a part of harmonic analysis related to the theory of spaces of homogeneous type (see [3. Chapter 6] as an introduction to this theory) and enjoy continued interest. To be more specific let us mention that if we extend a fundamental theory of Calderón-Zygmund singular integral operators to a more abstract setting, it turns out that the essential arguments are measure theoretic rather than Fourier analytic. The fundamental notion here is that of a space of homogeneous type which is, first of all, a quasi-metric space equipped in addition with a regular Borel measure that respects the quasi-metric in an appropriate way.

\section{MAin RESUlt}

Let $(X, \rho)$ be a quasi-metric space. Given $p, 0<p \leq 1$, define $d_{p}: X \times X \rightarrow$ $[0, \infty)$ by letting

$$
d_{p}(x, y)=\inf \left\{\sum_{j=1}^{n} \rho\left(x_{j-1}, x_{j}\right)^{p}: x=x_{0}, x_{1}, \ldots, x_{n}=y, \quad n \geq 1\right\} .
$$

Clearly, $d_{p}$ is symmetric and satisfies the triangle inequality

$$
d_{p}(x, y) \leq d_{p}(x, z)+d_{p}(z, y), \quad x, y, z \in X ;
$$

in addition, $d_{p} \leq \rho^{p}$. It is reasonable to refer to this process of producing $d_{p}$ from $\rho$ as the $p$-chain approach. It was shown in [1] that with $p$ chosen properly, $d_{p}(x, y) \neq 0$ for $x \neq y$; thus $d_{p}$ becomes a metric, in fact equivalent to $\rho^{p}$.

A similar approach in the context of quasi-normed spaces is known; see [2], 10], [7] and [9. Although the argument which is presented in [9] to prove a "quasinormed" result analogous to that from the proposition below doesn't seem to be directly applicable in the quasi-metric case, it gives a hint about how $p$ should be chosen.

Proposition. Let $(X, \rho)$ be a quasi-metric space and let $0<p \leq 1$ be given by $(2 K)^{p}=2$. Then $d_{p}$ obtained from $\rho$ by the $p$-chain approach is a metric on $X$ 
equivalent to $\rho^{p}$. In other words, $\tilde{\rho}=\tilde{\rho}(p)=d_{p}^{1 / p}$ is a quasi-metric on $X$ equivalent to $\rho$ and satisfying, in addition, the so-called p-triangle inequality

$$
\tilde{\rho}(x, y) \leq\left(\tilde{\rho}(x, z)^{p}+\tilde{\rho}(z, y)^{p}\right)^{1 / p}, \quad x, y, z \in X .
$$

The same conclusions hold if $\rho$ satisfies (i), (ii) and (iii)' with $K_{o} \geq 2$ and if $0<p \leq 1$ is then determined by $K_{o}^{p}=2$.

Proof. Clearly, it is sufficient to consider the case where $\rho$ satisfies (iii)' with $K_{o} \geq 2$. It has already been mentioned that $d=d_{p}$ given by (2.1) is symmetric, satisfies the triangle inequality and verifies the left hand side of the inequalities

$$
d(x, y) \leq \rho(x, y)^{p} \leq 4 d(x, y), \quad x, y \in X .
$$

Showing the right hand side of (2.2) will complete the proof that $d$ is a metric (equivalent to $\rho^{p}$ ). Obviously, the statements concerning $\tilde{\rho}$ then follow.

We prove by induction on $n$ that for any given sequence of $n+1$ points $x=$ $x_{0}, x_{1}, \ldots, x_{n}=y, n \geq 2$,

$$
\rho(x, y)^{p} \leq 2\left(\rho\left(x_{0}, x_{1}\right)^{p}+2 \sum_{j=1}^{n-2} \rho\left(x_{j}, x_{j+1}\right)^{p}+\rho\left(x_{n-1}, x_{n}\right)^{p}\right)
$$

(if $n=2$, then the middle term on the right hand side of (2.3) is absent). Consequently, $\rho(x, y)^{p} \leq 4 d(x, y)$ follows.

If $n=2$ and three points $x, x_{1}, y$ are given, then using $K_{o}^{p}=2$ gives

$$
\begin{aligned}
\rho(x, y)^{p} & \leq K_{o}^{p} \max \left\{\rho\left(x, x_{1}\right)^{p}, \rho\left(x_{1}, y\right)^{p}\right\} \\
& =2 \max \left\{\rho\left(x, x_{1}\right)^{p}, \rho\left(x_{1}, y\right)^{p}\right\} .
\end{aligned}
$$

Observe that, as a consequence, we obtain the starting point for the induction. Assume now that the induction hypothesis holds, (i.e. (2.3) is satisfied), and consider a sequence of $n+2$ points $x=x_{0}, x_{1}, \ldots, x_{n+1}=y$. Let $m$ be the largest number among $\{0,1, \ldots, n\}$ with the property

$$
\rho(x, y)^{p} \leq 2 \rho\left(x_{m}, y\right)^{p} .
$$

Since $\rho(x, y)^{p} \leq 2 \max \left\{\rho\left(x, x_{m+1}\right)^{p}, \rho\left(x_{m+1}, y\right)^{p}\right\}$, therefore

$$
\rho(x, y)^{p} \leq 2 \rho\left(x, x_{m+1}\right)^{p}
$$

(this is clear if $m \leq n-1$ and obvious for $m=n$ ). Combining (2.4) and (2.5) gives

$$
\begin{aligned}
\rho(x, y)^{p} & \leq 2 \min \left\{\rho\left(x, x_{m+1}\right)^{p}, \rho\left(x_{m}, y\right)^{p}\right\} \\
& \leq \rho\left(x, x_{m+1}\right)^{p}+\rho\left(x_{m}, y\right)^{p} .
\end{aligned}
$$

If it happens that $m=0$ or $m=n$, then the first inequality above readily gives the required conclusion, i.e. (2.3) with $n$ replaced by $n+1$, and there is actually no need to invoke the induction hypothesis. Assume therefore that $1 \leq m \leq n-1$. Then, applying the induction hypothesis to the sequences $x=x_{0}, x_{1}, \ldots, x_{m+1}$ and 
$x_{m}, x_{m+1}, \ldots, x_{n+1}=y$ (both of length $\left.\leq n+1\right)$ gives

$$
\begin{aligned}
\rho(x, y)^{p} \leq & \rho\left(x, x_{m+1}\right)^{p}+\rho\left(x_{m}, y\right)^{p} \\
\leq & 2\left(\rho\left(x_{0}, x_{1}\right)^{p}+2 \sum_{j=1}^{m-1} \rho\left(x_{j}, x_{j+1}\right)^{p}+\rho\left(x_{m}, x_{m+1}\right)^{p}\right) \\
& +2\left(\rho\left(x_{m}, x_{m+1}\right)^{p}+2 \sum_{j=m+1}^{n-1} \rho\left(x_{j}, x_{j+1}\right)^{p}+\rho\left(x_{n}, x_{n+1}\right)^{p}\right) \\
= & 2\left(\rho\left(x_{0}, x_{1}\right)^{p}+2 \sum_{j=1}^{n-1} \rho\left(x_{j}, x_{j+1}\right)^{p}+\rho\left(x_{n}, x_{n+1}\right)^{p}\right) .
\end{aligned}
$$

This completes the induction step and thus the proof of the Proposition.

If $(X, \rho)$ is a quasi-metric space, then $\mathcal{F}_{\rho}$, the topology in $X$ induced by $\rho$, is canonically defined by means of the theory of uniform structures; in case $\rho$ is a metric this procedure leads to the usual metric topology in $X$. We refer the reader to the monograph [4, where in Chapter 8 this way of introducing a topology is discussed.

The uniform structure $U_{\rho}$ generated by $\rho$ is defined to consist of all subsets $V \subset X \times X$, symmetric in the sense that $(x, y) \in V$ if and only if $(y, x) \in V$ and containing a set of the form $R_{\epsilon}=\{(x, y): \rho(x, y)<\epsilon\}$ for some $\epsilon>0$ (in particular $V$ contains the diagonal $\{(x, x): x \in X\}$ ). Since the countable family $\left\{R_{1 / n}\right\}_{n \geq 1}$ is a basis for the uniform structure $U_{\rho}$, it follows from a general result (see [4, Chapter 8, Theorem 9]) that the topology $\mathcal{F}_{\rho}$ generated by $U_{\rho}$ in $X$ is metrizable.

Given $r>0$ and $x \in X$, let

$$
B(x, r)=\{y \in X: \rho(x, y)<r\}
$$

be the quasi-metric ball related to $\rho$ of radius $r$ and with center $x$. According to the procedure of defining a topology by means of a uniform structure (see [4, Chapter 8]), in this case $G \subset X$ is defined to be open, i.e. $G \in \mathcal{F}_{\rho}$, if and only if for every $x \in G$ there exists $r>0$ such that $B(x, r) \subset G$ (at this point one easily checks directly that the topology axioms are satisfied for such a definition). It is clear that if $\rho_{1}$ is a quasi-metric equivalent to $\rho$, then $\mathcal{F}_{\rho_{1}}=\mathcal{F}_{\rho}$; also, for any $a>0$, $\rho^{a}$ is a quasi-metric as well and $\mathcal{F}_{\rho^{a}}=\mathcal{F}_{\rho}$. Thus the Proposition furnishes a direct argument showing that $\mathcal{F}_{\rho}$ is metrizable [1, 8 .

The quasi-metric balls themselves need not be open (unless $\rho$ is a genuine metric) as the following simple example shows.

Example. Let $X=\{0,1,2, \ldots\}$. Given $\epsilon>0$, we define $\rho=\rho_{\epsilon}$ on $X \times X$ in the following way. For $0 \leq n<m$, we set $\rho(n, m)$ as

$$
\begin{array}{cl}
\rho(0,1)=1, & \rho(0, m)=1+\epsilon \quad \text { if } m \geq 2, \\
\rho(1, m)=\frac{1}{m}, & \rho(n, m)=\frac{1}{n}+\frac{1}{m} \quad \text { if } n \geq 2 .
\end{array}
$$

We then extend $\rho$ onto $X \times X$ by putting $\rho(n, n)=0$ for any $n \geq 0$ and $\rho(n, m)=$ $\rho(m, n)$ if $0 \leq m<n$. We will show that

$$
\rho(k, n) \leq(1+\epsilon)(\rho(k, m)+\rho(m, n)), \quad k, m, n \in X,
$$


and thus $\rho$ is a quasi-metric with $K=1+\epsilon$. It is clear that it suffices to check (2.6) for pairwise distinct $k, m, n$ only. Let $L$ and $R$ denote the left and the right hand sides of the inequality (2.6), respectively. If one of $k, n, m$ is 0 , then $L \leq 1+\epsilon \leq R$. If none of $k, m, n$ are 0 , then we consider subcases. First, assume 1 appears among $k, m, n$. If $k=1$, then $L=\frac{1}{n}$ while $R=(1+\epsilon)\left(\frac{2}{m}+\frac{1}{n}\right)$. Similarly if $n=1$. If $m=1$, then $L=\frac{1}{k}+\frac{1}{n}$ while $R=(1+\epsilon)\left(\frac{1}{k}+\frac{1}{n}\right)$. Next, assume 1 does not appear among $k, n, m$. Then $L=\frac{1}{k}+\frac{1}{n}$ and $R=(1+\epsilon)\left(\frac{1}{k}+\frac{2}{m}+\frac{1}{n}\right)$. This finishes our checking that $\rho$ is indeed a quasi-metric with constant $K=1+\epsilon$.

Now, note that $B(0,1+\epsilon / 2)=\{0,1\}$ while $B(1, \eta)$ contains infinitely many elements, for any $\eta>0$. Hence, none of $B(1, \eta)$ are contained in $B(0,1+\epsilon / 2)$, which shows that $B(0,1+\epsilon / 2)$ is not open.

The metric $d_{p}$, produced from $\rho=\rho_{\epsilon}$ by the $p$-chain approach, can be computed directly. Let $p$ be defined as in the Proposition, with $1+\epsilon$ in place of $K$. It is not hard to see that with the notation $x_{0}=\left((1+\epsilon)^{p}-1\right)^{-1 / p}, d_{p}$ is given by

$$
d_{p}(k, n)= \begin{cases}1+\max \{k, n\}^{-p}, & \text { if } \min \{k, n\}=0 \text { and } \max \{k, n\}>x_{0}, \\ \rho(k, n)^{p}, & \text { otherwise. }\end{cases}
$$

It is easily seen that if for a quasi-metric space $(X, \rho)$ there exists a function $K(\epsilon), \epsilon>0$, such that $K(\epsilon) \rightarrow 1$ as $\epsilon \rightarrow 0^{+}$, and

$$
\forall x, y, z \in X \quad \rho(y, z)<\epsilon \rho(x, y) \Rightarrow \rho(x, z) \leq K(\epsilon)(\rho(x, y)+\rho(y, z)),
$$

then the quasi-metric balls $B(x, r), x \in X, r>0$, are open sets in the topology $\mathcal{F}_{\rho}$.

In particular, if a quasi-metric $\rho$ which, for some $p, 0<p \leq 1$, satisfies the $p$-triangle inequality

$$
\rho(x, z) \leq\left(\rho(x, y)^{p}+\rho(y, z)^{p}\right)^{1 / p}, \quad x, y, z \in X
$$

then $K(\epsilon)=\left(1+\epsilon^{p}\right)^{1 / p}$ is appropriate. Consequently, a quasi-metric space $(X, \rho)$ with $\rho$ satisfying (2.8) has all its quasi-metric balls open; hence this also happens for the quasi-metric $\tilde{\rho}=\tilde{\rho}(p)$ from the Proposition.

It is clear that the quasi-metric space $(X, \rho)$ from the Example must fail to satisfy (2.8) for any $p, 0<p \leq 1$. To see this by a direct argument note that for $x=0, y=1$ and $z=n \geq 2$ we have $\rho(0, n)=1+\epsilon$, while

$$
\left(\rho(0,1)^{p}+\rho(1, n)^{p}\right)^{1 / p}=\left(1+n^{-p}\right)^{1 / p} \rightarrow 1, \quad \text { as } n \rightarrow \infty .
$$

Given a quasi-metric space $(X, \rho)$, let $K(\rho)$ denote the smallest constant $K$ for which (iii) holds, and let $p(\rho)$ denote the largest $p \in(0,1]$ for which (2.8) holds; if such $p$ does not exist, then we set $p(\rho)=0$. For instance, for the quasi-metric $\rho_{\epsilon}$ from the example we have $K\left(\rho_{\epsilon}\right)=1+\epsilon$ and $p\left(\rho_{\epsilon}\right)=0$. Also, let $\tilde{p}(\rho)$ denote the supremum of the set of $p \in(0,1]$ with the property that there exists a quasi-metric $\tilde{\rho}$ equivalent to $\rho$ and such that (2.8) is satisfied with $\tilde{\rho}$ replacing $\rho$. Equivalently,

$$
\tilde{p}(\rho)=\sup \left\{p \in(0,1]: d_{p} \quad \text { is a metric equivalent to } \rho^{p}\right\} .
$$

It follows from the Proposition that for any given $(X, \rho)$ one has

$$
\tilde{p}(\rho) \geq \frac{1}{\log _{2}(2 K(\rho))}
$$


Note that in [1] the weaker estimate

$$
\tilde{p}(\rho) \geq \frac{1}{\left(\log _{2}(3 K(\rho))\right)^{2}}
$$

was proved. A simple example of the usual $\ell^{p}$ spaces, $0<p<1$, shows that the estimate (2.9) cannot, in general, be improved.

It can happen, however, that the inequality (2.9) is strict. In fact, for the quasimetric $\rho_{\epsilon}$ from the above example one can apply the $p$-chain approach for any $0<p \leq 1$ and obtain a metric. Thus for this rather pathological example, actually $\tilde{p}(\rho)=1$.

\section{ACKNowledgements}

The authors would like to thank Professor Roberto Macías for pointing out to them reference [1] and the referee for bringing to their attention references [6] and 7] as well as for suggesting numerous improvements in the presentation.

\section{REFERENCES}

[1] H. Aimar, B. Iaffei and L. Nitti, On the Macías-Segovia metrization of quasi-metric spaces, Revista U. Mat. Argentina 41 (1998), 67-75. MR.1700292 (2000e:54019)

[2] T. Aoki, Locally bounded linear topological spaces, Proc. Imp. Acad. Tokyo 18 (1942), 588594. MR0014182 (7:250d)

[3] M. Christ, Lectures on Singular Integral Operators, CBMS Reg. Conf. Ser. Math., vol. 77, Amer. Math. Soc., Providence, RI, 1990. MR1104656 (92f:42021)

[4] R. Engelking, General Topology, Heldermann Verlag, Berlin, 1989. MR.1039321 (91c:54001)

[5] A. H. Frink, Distance functions and the metrization problem, Bull. Amer. Math. Soc. 43 (1937), 133-142. MR.1563501

[6] J. Heinonen, Lectures on Analysis on Metric Spaces, Universitext, Springer-Verlag, New York, 2001. MR.1800917 (2002c:30028)

[7] N. J. Kalton, N. T. Peck, and J. W. Roberts, An F-space Sampler, London Math. Society Lecture Note Series, vol. 89, Cambridge University Press, Cambridge, 1984. MR808777 (87c:46002)

[8] R. A. Macías and C. Segovia, Lipschitz functions on spaces of homogeneous type, Adv. in Math. 33 (1979), 257-270. MR.546295 (81c:32017a)

[9] J. Peetre and G. Sparr, Interpolation of normed abelian groups, Ann. Mat. Pura Appl. (4) 92 (1972), 217-262. MR0322529 (48:891)

[10] S. Rolewicz, On a certain class of linear metric spaces, Bull. Acad. Polon. Sci. Cl. III 5 (1957), 471-473. MR0088682(19:562d)

Instytut Matematyczny, Uniwersytet WrocŁawski, Pl. Grunwaldzki 2/4, 50-384 Wroceaw, Poland

E-mail address: mpal@math.uni.wroc.pl

Instytut Matematyki i Informatyki, Politechnika WrocŁawska, Wyb. Wyspiańskiego 27, 50-370 WrocŁaw, Poland - and - Katedra Matematyki i Zastosowań Informatyki, Politechnika Opolska, ul. MikoŁajczyka 5, 45-271 Opole, Poland

E-mail address: Krzysztof.Stempak@pwr.wroc.pl 